\title{
Prevalence of Altered Passive Eruption in Orthodontically Treated and Untreated Patients
}

Jose Nart, * Neus Carrió, * Cristina Valles, ${ }^{*}$ Carols Solís-Moreno, ${ }^{*}$ Maria Nart, ${ }^{\dagger}$ Ramon Reñé, ${ }^{\ddagger}$ Cristina Esquinas, $\$$ and Andreu Puigdollers"

Background: Some authors have reported that after orthodontic treatment (OT), a "gummy smile" might develop. Nevertheless, there are no studies in the literature that investigate whether OT increases the presence of altered passive eruption (APE). The primary aim of this cross-sectional study is to evaluate the prevalence of APE after OT (OT group) and compare it with patients who never received OT (control group). A secondary aim is to identify which variables are related to APE.

Methods: The study population consisted of 190 patients (95 patients each in the control and OT groups), providing 1,140 anterior teeth for the clinical examination. The following clinical parameters were assessed: presence or absence of APE, clinical crown length, and gingival biotype, which was divided into three categories: thin-scalloped, thick-flat, and thick-scalloped.

Results: Twenty-eight patients (29.5\%) were diagnosed with APE in the control group and 40 (42.1\%) in the OT group, although this difference was not statistically significant $(P=0.07)$. Furthermore, $34(75.6 \%)$ patients with thickflat biotype were diagnosed with APE, whereas 30 (31.3\%) and four $(8.2 \%)$ with thick-scalloped and thin-scalloped biotypes, respectively, had APE. These differences were statistically significant $(P<0.001)$.

Conclusions: It was concluded that: 1$)$ the prevalence of APE is higher after OT but not to a statistically significant degree and 2) APE is more common in individuals with a thickflat gingival biotype. J Periodontol 2014;85:e348-e353.

\section{KEY WORDS}

Cemento-enamel junction; crown lengthening; orthodontics; periodontics; surgery, plastic; tooth eruption.

* Department of Periodontology, School of Dentistry, International University of Catalunya, Barcelona, Spain.

$\dagger$ Department of Periodontology, University of Granada, Granada, Spain.

\# Private practice, Mollerussa, Lleida, Spain.

$\S$ Department of Research, School of Dentistry, International University of Catalunya.

\| Department of Orthodontics, School of Dentistry, International University of Catalunya.
I $n$ dentistry, esthetics is one of the major concerns for both practitioners and patients. An esthetically pleasing smile is defined as one in which the size, shape, position, and color of the teeth are in harmony with surrounding gingival soft tissue, the lips, and finally, the face. ${ }^{1}$ As classically described, the ideal smile includes a gingival display of approximately 1 to $2 \mathrm{~mm} .^{2}$ However, some patients show $>2 \mathrm{~mm}$ of gingival tissue. Although this situation is not pathologic, it can produce a poor esthetic effect known as a "gummy smile."3 Excessive gingival display or gummy smile may be the result of a short upper lip, hypermobile lip, vertical maxillary excess, anterior overeruption, wear and compensatory eruption, altered active eruption, and altered passive eruption (APE) ${ }^{4}$

APE is a clinical condition wherein "the gingiva in the adult is located incisal to the cervical convexity of the crown and removed from the cemento-enamel junction (CEJ) of the tooth." 5 APE has also been referred to as retarded passive eruption, ${ }^{6}$ incomplete passive eruption, ${ }^{7}$ and delayed passive eruption. ${ }^{8,9}$ Regardless of its name, APE can lead to clinical crowns that appear square in shape and can be perceived as unesthetic. ${ }^{6}$

Gottlieb and Orban ${ }^{10}$ described two phases of tooth eruption. The active phase of eruption is defined by emerging motion of the tooth on the occlusal direction until the tooth reaches the occlusal 
plane of its antagonist. This process is accompanied by passive eruption, which is the apical migration of the soft tissues, with gradual exposure of the crown of the tooth. Classically, the passive phase has been divided into four stages according to the location of the dentogingival junction (DGJ) with respect to the CEJ. ${ }^{11}$ In Phase I, the DGJ is located on the enamel; in Phase II, the DGJ is located on enamel and cementum; in Phase III, the DGJ is located entirely on the cementum; and in Phase IV, the DGJ is on cementum, and the root surface is exposed. At present, only the first stage is considered to be physiologic, while the remaining three are a consequence of pathologic periodontal breakdown processes.

Evidence to date fails to clarify the causes and mechanisms that may lead to APE, although a few studies have related such mechanisms to the morphology adopted by the coronal periodontium. ${ }^{12}$

Kokich $^{3}$ suggested that there are three unesthetic situations that may develop during orthodontic treatment (OT): 1) the gummy smile; 2) gingival margin discrepancies; and 3) the "missing papilla." Furthermore, Keim ${ }^{13}$ reported that, "the resulting gummy smile in these cases is most recognizable by the fact that the patient presents with shortened clinical crowns and gingival tissue that is obviously thicker than normal in the labiolingual dimension."

Nevertheless, to the best of the present authors' knowledge, there are no studies in the literature that investigate whether OT increases the presence of APE. Therefore, the primary aim of this cross-sectional study is to evaluate the prevalence of APE after OT and compare it with patients who never received OT. A secondary aim is to identify which variables are related to APE.

\section{MATERIALS AND METHODS}

\section{Study Design and Patient Selection}

A total of 190 consecutive patients ( 71 males and 119 females, aged 22 to 38 years; mean age: $26.41+3.67$ years) referred to the Department of Periodontology at International University of Catalunya (UIC), Barcelona, Spain, from February 2011 to December 2013 were included in this cross-sectional study. Patients were classified in two groups: those who finished OT (full fixed appliances) at the Department of Orthodontics (UIC, Barcelona, Spain) before age 20 (OT group) and those who never received OT (control group).

In the control group, the mean age of 95 patients (36 males and 59 females) was $26.47 \pm 3.83$ years (range: 22 to 38 years), and in the OT group the mean age of 95 patients ( 35 males and 60 females) was $26.34 \pm 3.51$ years (range: 22 to 36 years).

Patients were informed about the nature of the study and were required to provide signed informed consent. The UIC's Ethics Committee approved the study. Individuals were selected according to the following inclusion criteria: 1) systemically healthy; 2) non-smoking; 3) all maxillary front teeth present from canine to canine; 4) no evidence of attachment loss, gingival overgrowth/hyperplasia, or inflammation; 5) no history of periodontal surgery; 6) not pregnant or lactating; 7) CEJ detectable with periodontal probe; and 8) no alterations of the incisal edge tooth such as restorations, traumatic injury, or attrition. Because the length of the clinical crown has shown statistically significant changes up to 19 years, ${ }^{14}$ only individuals aged $\geq 20$ years were included in the study. Patients were excluded from the study if they were taking drugs causing gingival enlargement, received OT after the age of 20 , or received interceptive OT. Because of the limited ethnic diversity, only data from whites were analyzed.

\section{Data Collection and Clinical Measurements}

An interview was conducted to obtain demographic characteristics of the study population (age and sex). In addition, data of other variables were collected such as OT, being the main independent variable, duration of OT, clinical crown length, and gingival biotype. The primary outcome variable was the presence of APE.

Clinical parameters (presence or absence of APE, clinical crown length, and gingival biotype) were assessed by the same calibrated examiner (JN) at the mid-buccal site of the six maxillary anterior teeth. The examiner was masked to whether patients received OT.

Absence of APE was determined with a periodontal probe ${ }^{\text {Il }}$ to the nearest $0.5 \mathrm{~mm}$ if the distance from the gingival margin to the CEJ was 0.5 to $2 \mathrm{~mm} .{ }^{15} \mathrm{~A}$ diagnosis of APE was established if the distance from the gingival margin to the CEJ was $>2 \mathrm{~mm}$ in $\geq 2$ of the six maxillary anterior teeth because of esthetic relevance. Local anesthesia was delivered if the patient felt uncomfortable.

Clinical crown length was defined as the distance from the gingival margin to the incisal edge of the crown for each study tooth. Measurements were made on stone casts to the nearest $0.1 \mathrm{~mm}$ by using a caliper." Results obtained from both central incisors were averaged, as well as for lateral incisors and canines.

Gingival biotype was divided into three categories: thin-scalloped, thick-flat, and thick-scalloped. ${ }^{16}$ The determination of the gingival thickness was based on the transparency of the same periodontal probe through the gingival margin. ${ }^{16,17}$

II PCP-UNC 15, Hu-Friedy, Chicago, IL.

\# BG, Hu-Friedy. 


\section{Intra-Examiner Repeatability}

The intra-examiner repeatability of the clinician was conducted by measuring the distance from the gingival margin to the CEJ in 10 volunteers, not involved in the study, on two occasions 24 hours apart. Calibration was accepted if $90 \%$ of the recordings could be reproduced within a difference of $0.5 \mathrm{~mm}$.

\section{Sample Size Calculation}

According to Volchansky and Cleaton-Jones, ${ }^{9}$ the prevalence of APE is $12 \%$. The hypothesis was that the prevalence of APE in orthodontic patients is greater, $\approx 25 \%$ to $30 \%$. Accepting an $\alpha$ error of $5 \%$ and a statistical power of $90 \%$ in a bilateral contrast, 190 individuals (95 patients in each group) were needed to detect a minimum difference of $15 \%$ between groups for statistical significance. The arcsin approximation was used.

\section{Statistical Analyses}

A patient-level analysis was performed for presence or absence of APE and gingival biotype, whereas a cluster-level analysis was carried out for the clinical crown length. Descriptive statistics were expressed as mean \pm SD and percentage for continuous and categorical data, respectively. The comparisons between groups were performed using the $\chi^{2}$ test (exact Fisher test with observed frequencies $<5$ ) for categorical variables or Kendall $\tau$-c for trend analysis, whereas continuous variables were tested using $t$ test (Mann-Whitney $U$ test if the variables were not normally distributed). Crude odds ratios (ORs) were calculated in some relationships. Finally, a stepwise multivariate logistic regression model was performed to identify which variables were related to APE. Relevant clinical variables were included in the final model (study group, age, sex, duration of OT, and gingival biotype) as independent variables with a significant univariate association $(P<0.3) .{ }^{18} P<0.05$ was considered to be statistically significant. All statistical analyses were performed using a statistical software package. **

\section{RESULTS}

A total of 190 patients were enrolled in the study (95 patients in each group) providing 1,140 anterior teeth for the clinical examination (Fig. 1). No statistically significant differences were found between the groups for age $(P=0.80)$ or sex $(P=0.88)$.

Twenty-eight patients $(29.5 \%)$ were diagnosed with APE in the control group and 40 (42.1\%) in the OT group, although this difference was not statistically significant $(P=0.07)$ (Table 1$)$. When comparisons were made between males and females, no statistically significant differences in the prevalence of APE were observed (38.0\% and 34.5\%, respectively; $P=0.62$ ). Furthermore, it was observed that OT did
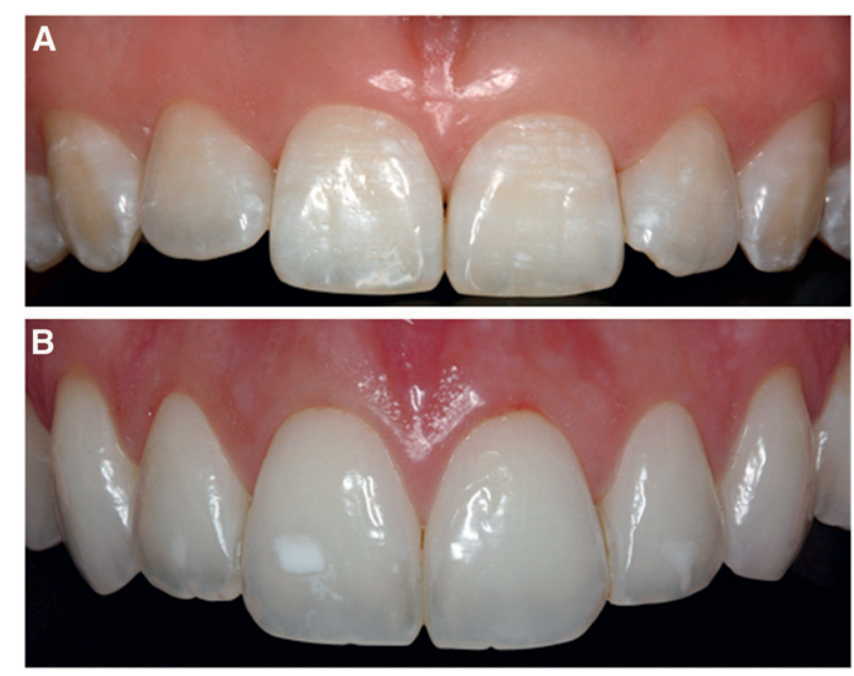

Figure 1 .

APE versus normal eruption. A) APE. B) Normal eruption.

Table I.

\section{Prevalence of APE ( $n[\%])$ in Control and OT Groups}

\begin{tabular}{lccc}
\hline & $\begin{array}{c}\text { Control } \\
\text { Group }(n=95)\end{array}$ & $\begin{array}{c}\text { OT Group } \\
(n=95)\end{array}$ & $P$ \\
\hline APE & $28(29.5)$ & $40(42.1)$ & $0.07^{*}$ \\
Non-APE & $67(70.5)$ & $55(57.9)$ & \\
\hline
\end{tabular}

* No statistically significant difference $(P>0.05)$.

not increase the probability of APE (crude OR = 1.740; 95\% confidence interval [CI] 0.955 to 3.172).

The mean starting age of the OT was $13.80 \pm 2.55$ years and the mean finishing age was $16.15 \pm 2.45$ years. The mean duration of OT was $2.4 \pm 1.0$ years (range 1 to 7 ). No relationship was observed between the presence of APE and duration of OT $(P=0.78)$.

In the control group, the mean length of the clinical crowns was $9.85 \pm 1.21 \mathrm{~mm}$ for canines, $8.67 \pm 1.00$ $\mathrm{mm}$ for lateral incisors, and $10.29 \pm 1.03 \mathrm{~mm}$ for central incisors. The corresponding results for the OT group were $9.69 \pm 1.00,8.52 \pm 0.91$, and $10.20 \pm$ $0.76 \mathrm{~mm}$. No statistically significant differences were found between the groups for canines $(P=0.34)$, lateral incisors $(P=0.28)$, and central incisors $(P=$ 0.50) (Table 2).

Finally, thick-flat, thick-scalloped, and thin-scalloped gingival biotype was present in 45, 96, and 49 patients. Results from the bivariate analysis only showed a statistically significant association between APE and gingival biotype (age, sex, and duration of

* * SPSS v.17.0, IBM, Chicago, IL. 
OT were not related to APE). Thirty-four (75.6\%) individuals who had thick-flat biotype were diagnosed with APE, whereas 30 (31.3\%) and four $(8.2 \%)$ with thick-scalloped and thin-scalloped biotypes, respectively, had APE. These differences were statistically significant $(P<0.001)$ (Table 3$)$. Moreover, a linear trend was observed toward lower prevalence of APE in patients with a thin-scalloped gingival biotype $(P<0.001)$. Comparing control and OT groups, similar results were obtained. In the control group, the percentage of patients with thickflat biotype with APE was significantly greater than with thick-scalloped and thin-scalloped biotypes (60.9\%, 28.3\%, and 3.8\%, respectively; $P<0.001)$. The corresponding results for the OT group were 90.9\%, 34.0\%, and 13.0\% ( $P<0.001)$.

To further investigate which variables were related to APE, a stepwise multivariate logistic regression model was constructed (Table 4). The independent variables were study group, age, sex, duration of OT, and gingival biotype (the reference category was thick-flat biotype), whereas APE was considered as a dependent variable. Only gingival biotype was related to APE, and patients with a thick-scalloped or thin gingival biotype were less likely to have APE than those with thick-flat biotype $(\mathrm{OR}=0.052 ; 95 \%$

\section{Table 2.}

Comparison of Clinical Crown Length ( $\mathrm{mm}$ ) of Central Incisors, Lateral Incisors, and Canines in OT and Control Groups (mean \pm SD)

\begin{tabular}{lccc}
\hline Teeth & OT Group & Control Group & $P$ \\
\hline Central incisors & $10.20 \pm 0.76$ & $10.29 \pm 1.03$ & $0.50^{*}$ \\
Lateral incisors & $8.52 \pm 0.91$ & $8.67 \pm 1.00$ & $0.28^{*}$ \\
Canines & $9.69 \pm 1.00$ & $9.85 \pm 1.21$ & $0.34 *$ \\
\hline
\end{tabular}

* No statistically significant difference $(P>0.05)$.

Table 3.

Comparison of Gingival Biotype (n [\%]) for Individuals With and Without APE in Control and OT Groups

\begin{tabular}{lcccc}
\hline & $\begin{array}{c}\text { Thick-Flat } \\
(\mathrm{n}=45)\end{array}$ & $\begin{array}{c}\text { Thick-Scalloped } \\
(\mathrm{n}=96)\end{array}$ & $\begin{array}{c}\text { Thin-Scalloped } \\
(\mathrm{n}=49)\end{array}$ & $P$ \\
\hline APE & $34(75.6)$ & $30(31.3)$ & $4(8.2)$ & \\
Non-APE & II $(24.4)$ & $66(68.7)$ & $45(91.8)$ & $<0.00$ । * \\
\hline
\end{tabular}

* Statistically significant difference $(P<0.05)$.
CI 0.011 to 0.247 ; and $0.015 ; 0.002$ to 0.100 , respectively).

\section{DISCUSSION}

The results reported in this study demonstrate that after OT, the prevalence of APE is higher than in individuals who never received OT. Twenty-eight patients $(29.5 \%)$ were diagnosed with APE in the control group and $40(42.1 \%)$ in the OT group; however, these differences were not statistically significant $(P=0.07)$. These results are in agreement with the clinical impressions of some authors, ${ }^{3,13}$ who reported that after OT patients often develop a gummy smile.

Data regarding prevalence are helpful because they allow practitioners to know how often they should be observing a given condition. In this study, the prevalence of APE was $35.8 \%$ (29.5\% for the control group and $42.1 \%$ for the OT group). However, based on a series of 1,025 patients with a mean age of $24.2 \pm 6.2$ years, Volchansky and Cleaton-Jones 9 recorded a $12.1 \%$ prevalence of APE. One possible explanation for this could be the different diagnostic criteria used for APE. Volchansky and CleatonJones ${ }^{9}$ diagnosed APE "when the gingival margin was coronal to the CEJ at a level approximating the maximum convexity of the buccal or labial aspect of the tooth and associated with pseudo-pocketing." It has been described that the curvature of the cervical line in a maxillary central incisor is 3 to $4 \mathrm{~mm} .{ }^{19}$ Thus, the prevalence of APE observed by Volchansky and Cleaton-Jones ${ }^{9}$ included only those teeth with a gingival margin positioned 3 to $4 \mathrm{~mm}$ over the CEJ. In the present study, a diagnosis of APE was established if the distance from the gingival margin to the CEJ was $>2 \mathrm{~mm}$ in $\geq 2$ of the six anterior teeth.

It should be noted that the prevalence of APE in the adult population has been little studied to date, possibly because of the lack of clear diagnostic criteria. There are some clinical criteria such as short clinical crown and flat gingival architecture that are commonly used for the diagnosis of APE. However, these criteria are subjective and may be related to anatomic structures. ${ }^{20}$ Therefore, to carry out a clinical diagnosis, it is necessary to search for the position of the CEJ with a probe. If the distance from the gingival margin to the CEJ is 0.5 to $2 \mathrm{~mm},{ }^{15}$ a greater distance of $2 \mathrm{~mm}$ could be used as a diagnostic criterion of APE. On the other hand, AlpisteIllueca $^{21}$ showed that when the left maxillary central incisor had APE, the lengths of the clinical crowns of all teeth in the upper anterior sextant were significantly smaller than in the rest of the series. These results suggest that APE usually affects all second sextant teeth. 
Table 4.

\section{Univariate and Stepwise Logistic Regression Model of APE}

\begin{tabular}{|c|c|c|c|c|}
\hline Variable & Crude OR $(95 \% \mathrm{Cl})$ & $P$ & OR $(95 \% \mathrm{Cl}) *$ & $P$ \\
\hline OT group versus control & 1.740 (0.955 to 3.172$)$ & 0.07 & - & - \\
\hline Age (years) & 0.975 (0.898 to 1.058$)$ & 0.19 & - & - \\
\hline Sex (female) & 0.857 (0.465 to I.577) & 0.24 & - & - \\
\hline \multicolumn{5}{|l|}{ Biotype } \\
\hline Thick-flat ${ }^{\dagger}$ & 1 & & I & \\
\hline Thick-scalloped & 0.147 (0.066 to 0.329 ) & $<0.001$ & 0.052 (0.01। to 0.247$)$ & $<0.001$ \\
\hline Thin-scalloped & 0.029 (0.008 to 0.098$)$ & $<0.001$ & $0.015(0.002$ to 0.100$)$ & $<0.001$ \\
\hline Duration of OT (years) & 1.057 (0.715 to 1.563$)$ & 0.78 & - & - \\
\hline
\end{tabular}

* Stepwise multivariate logistic regression model.

$\dagger$ Reference category.

According to Sterrett et al., ${ }^{6}$ the ideal crown length for maxillary anterior teeth was defined as $9.79 \mathrm{~mm}$ for centrals, $8.24 \mathrm{~mm}$ for laterals, and $9.47 \mathrm{~mm}$ for canines. When comparing mean observed values of the maxillary anterior clinical crowns to those presented by Sterrett et al., lengths were similar. ${ }^{6}$

Despite no statistically significant differences being seen for clinical crown lengths between control and OT groups, it should be noted that there was a tendency toward higher clinical crown lengths for teeth in the control group. If one considers that a short clinical crown is one of the clinical criteria of APE, these results could probably explain the clinical impressions of some authors, ${ }^{3,13}$ who reported that after OT a gummy smile may develop.

Konikoff et al. ${ }^{22}$ evaluated the clinical crown length of the maxillary anterior teeth pre- and postorthodontics (the day of bracket removal and 5 years after orthodontic completion). Immediately after OT, clinical crown length in maxillary central incisors did not change from pre- to postorthodontic values, whereas that in lateral incisors and canines increased. By the clinical examination $\geq 5$ years later, all maxillary anterior clinical crowns increased significantly in length. However, most patients included in their study were $<20$ years old $(34.5 \%$ of participants aged 8 to 15 years and $28.5 \%$ of volunteers aged 16 to 20 years), and the literature demonstrated that there is an increase in the clinical crown length of individuals up to 19 years. ${ }^{14}$ Clearly, passive eruption most likely occurred from the completion of orthodontics to the clinical examination 5 years later. In contrast, in the present study, all patients were 20 years or older.

Few studies have evaluated the possible causes of APE. A number of factors have been proposed, such as interocclusal interference on the part of soft tissues during the eruptive phase, a certain hereditary tendency in families with individuals presenting $\mathrm{APE}$, and the presence of thick and fibrotic gingival biotype that tends to migrate more slowly during the passive phase than thin gingival tissue. ${ }^{12,13}$

In this study, $75.6 \%$ of patients that had thick-flat biotype (short and wide teeth, a broad zone of keratinized tissue, and a flat, slightly scalloped gingival margin) were diagnosed with APE, whereas only $8.2 \%$ with thin-scalloped biotype had APE. The separate analysis of the two groups showed similar results. It is important to note that gingival biotype was, independently of age, sex, study group, and duration of OT, associated with APE.

Batista et al. ${ }^{23}$ used cone-beam computed tomography to diagnose and characterize the hard and soft tissue anatomic features of APE-affected teeth. The mean soft tissue thickness (measured $2 \mathrm{~mm}$ apical to the gingival margin) was $\geq 1 \mathrm{~mm}$ for canines, lateral incisors, and central incisors, and all individuals with APE presented features of a thick biotype with a flat gingival architecture. In the present study, the gingival biotype was determined based on the visibility of a periodontal probe through the gingival margin. Some studies ${ }^{17,24}$ have reported that transgingival probing is an adequately reliable and objective method to evaluate gingival biotype.

The major limitations of this study are the lack of evidence for the criteria used to diagnose APE at a patient-level analysis and the difficulty in the detection of the CEJ with a periodontal probe. However, this is the first study that evaluates which factors are related to APE. Further investigations are needed to confirm these findings.

\section{CONCLUSIONS}

It was concluded that: 1) the prevalence of APE is higher after OT but not to a statistically significant 
degree and 2) APE is more common in individuals with thick-flat gingival biotype.

\section{ACKNOWLEDGMENT}

The authors report no conflicts of interest related to this study.

\section{REFERENCES}

1. Moskowitz ME, Nayyar A. Determinants of dental esthetics: A rational for smile analysis and treatment. Compend Contin Educ Dent 1995;16:1164-1166.

2. Kokich VG, Nappen DL, Shapiro PA. Gingival contour and clinical crown length: Their effect on the esthetic appearance of maxillary anterior teeth. Am J Orthod 1984;86:89-94.

3. Kokich VG. Esthetics: The orthodontic-periodontic restorative connection. Semin Orthod 1996;2:21-30.

4. Robbins JW. Differential diagnosis and treatment of excess gingival display. Pract Periodontics Aesthet Dent 1999;11:265-272, quiz 273.

5. Goldman, HM, Cohen, DW. Periodontal Therapy, 4th ed. St. Louis, MO: Mosby; 1968:35-39.

6. Sterrett JD, Oliver T, Robinson F, Fortson W, Knaak B, Russell CM. Width/length ratios of normal clinical crowns of the maxillary anterior dentition in man. $J$ Clin Periodontol 1999;26:153-157.

7. Hempton TJ, Esrason F. Crown lengthening to facilitate restorative treatment in the presence of incomplete passive eruption. J Mass Dent Soc 1999;47: 17-22, 24.

8. Coslet JG, Vanarsdall R, Weisgold A. Diagnosis and classification of delayed passive eruption of the dentogingival junction in the adult. Alpha Omegan 1977;70: 24-28.

9. Volchansky A, Cleaton-Jones PE. Delayed passive eruption. A predisposing factor to Vincent's infection? J Dent Assoc S Afr 1974;29:291-294.

10. Gottlieb, B, Orban, B. Active and passive continuous eruption of teeth. J Dent Res 1933;13:214.

11. Gargiulo AW, Wentz FM, Orban B. Dimensions and relations of the dentogingival junction in humans. J Periodontol 1961;32:12-35.

12. Alpiste-Illueca F. Altered passive eruption (APE): A little-known clinical situation. Med Oral Patol Oral Cir Bucal 2011;16:100-104.

13. Keim RG. Aesthetics in clinical orthodontic-periodontic interactions. Periodontol 2000 2001;27:59-71.
14. Volchansky A, Cleaton-Jones P. Clinical crown height (length) - A review of published measurements. J Clin Periodontol 2001;28:1085-1090.

15. Ainamo J, Löe H. Anatomical characteristics of gingiva. A clinical and microscopic study of the free and attached gingiva. J Periodontol 1966;37:5-13.

16. De Rouck T, Eghbali R, Collys K, De Bruyn H, Cosyn J. The gingival biotype revisited: Transparency of the periodontal probe through the gingival margin as a method to discriminate thin from thick gingiva. J Clin Periodontol 2009;36:428-433.

17. Cook DR, Mealey BL, Verrett RG, et al. Relationship between clinical periodontal biotype and labial plate thickness: An in vivo study. Int $J$ Periodontics Restorative Dent 2011;31:345-354.

18. O'Gorman TW, Woolson RF. Variable selection to discriminate between two groups; stepwise logistic regression or stepwise discriminate analysis? Am Stat 1991;45:187-193.

19. Ash MM, Nelson SJ. Wheeler's Dental Anatomy, Physiology, and Occlusion, 8th ed. St. Louis: Saunders; 2003:159.

20. Levine RA, McGuire M. The diagnosis and treatment of the gummy smile. Compend Contin Educ Dent 1997; 18:757-62, 764; quiz 766.

21. Alpiste-Illueca F. Morphology and dimensions of the dentogingival unit in the altered passive eruption. Med Oral Patol Oral Cir Bucal 2012;17:814-820.

22. Konikoff BM, Johnson DC, Schenkein HA, Kwatra N, Waldrop TC. Clinical crown length of the maxillary anterior teeth preorthodontics and postorthodontics. $J$ Periodontol 2007;78:645-653.

23. Batista EL Jr., Moreira CC, Batista FC, de Oliveira RR, Pereira KK. Altered passive eruption diagnosis and treatment: A cone beam computed tomography-based reappraisal of the condition. J Clin Periodontol 2012; 39:1089-1096.

24. Kan JY, Morimoto T, Rungcharassaeng K, Roe P, Smith $\mathrm{DH}$. Gingival biotype assessment in the esthetic zone: Visual versus direct measurement. Int $J$ Periodontics Restorative Dent 2010;30:237-243.

Correspondence: Prof. Andreu Puigdollers, Department of Orthodontics, International University of Catalunya, C/ Josep Trueta s/n, Sant Cugat del Vallés 08195. Barcelona, Spain. Fax: 34-935042001; e-mail: apuigdollersp@gmail.com.

Submitted May 5, 2014; accepted for publication June 30, 2014. 